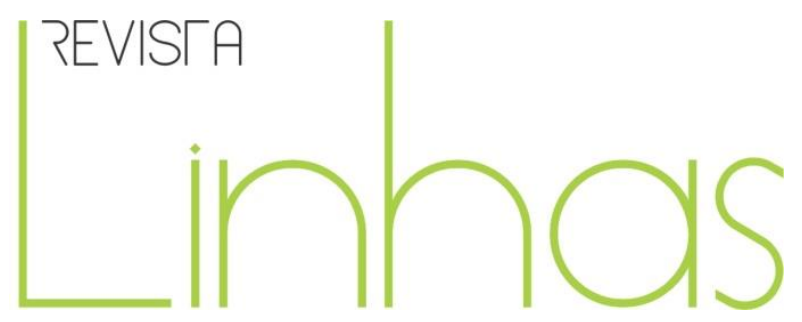

\title{
Arte y música en la educación en México: discursos, realidades y retos
}

\begin{abstract}
Resumen
En este artículo se presenta una revisión y análisis de las políticas educativas en México en materia de educación del arte y la música, en el marco de la Reforma Educativa de 2016, la cual en muy diversos sentidos ha sido controversial y no ha tenido la acogida esperada por los agentes educativos involucrados en el sistema educativo nacional y por la ciudadanía en general. Además, ante los recientes cambios políticos, el próximo gobierno federal presidido por Andrés Manuel López Obrador se ha encaminado a realizar una consulta nacional para la derogación de dicha reforma. Dada la incertidumbre actual en materia educativa, este análisis presenta el panorama actual de la educación artística, en pleno proceso de transición política. El análisis sugiere que tanto la legislación como los planes y programas de estudio muestran consistencia con el discurso político, orientado hacia el logro de una educación de calidad, con sustentos teóricos y fundamentos curriculares alineados a las tendencias internacionales en materia de educación. Sin embargo, la operacionalización del nuevo modelo educativo, en lo referente a la educación artística y musical, presenta problemas y retos sustanciales para su adecuada implementación, más aún enfrenta el reto de su propia permanencia ante este devenir histórico. El análisis de los programas de artes actuales revela un retroceso en el posicionamiento de la educación artística dentro del currículo, en comparación con anteriores planes de estudio. En qué medida este posicionamiento de la educación artística mejora, en virtud de la actual transición política, está aún por verse.
\end{abstract}

Palabras clave: Artes y Educación. Reforma Educativa. Planes y Programas de Estudio. Educación Musical. Políticas Educativas.

\author{
Patricia A. González-Moreno \\ Universidad Autónoma de \\ Chihuahua - México \\ pagonzalez@uach.mx
}

\author{
Para citar este artigo: \\ GONZÁLEZ-MORENO, Patricia A. Arte y música en la educación en México: discursos, realidades y \\ retos. Revista Linhas. Florianópolis, v. 20, n. 42, p. 36-62, jan./abr. 2019.
}

DOI: $10.5965 / 1984723820422019036$

http://dx.doi.org/10.5965/1984723820422019036 


\section{Arts and Music in Mexican Education: realities, and challenges}

Abstract

This article presents a review and analysis of educational policies in Mexico regarding art and music education, within the framework of the last Educational Reform, 2016, which in many different ways has been controversial and has not had the welcome expected by educational agents involved in the national education system and by the general public.In addition, before the recent political changes, the next federal government chaired by Andrés Manuel López Obrador, has been directed to conduct a national consultation to repeal this reform. Given the current uncertainty in education, this analysis presents the current panorama of artistic education, in the process of political transition. The analysis suggests that both the legislation and the national curriculum show consistency with the political discourse, oriented towards the achievement of quality education, with theoretical and curricular foundations aligned with international trends in education. However, the operationalization of the new educational model, in terms of arts and music education, presents substantial problems and challenges for its proper implementation, moreover, it faces the challenge of its own permanence through this historical transition. In addition, the analysis of the new arts curriculum shows a decline in the positioning of arts and music education within the curriculum, compared to previous curricula. To what extent the position of arts education within compulsory education improves, by virtue of the current political transition, remains to be seen.

Keywords: Art and Education. Educational Reform. Curriculum. Music Education. Educational Policies.

\section{Arte e música na educação no México: discursos, realidades e desafios}

\section{Resumo}

Neste artigo, apresenta-se uma revisão e análise das políticas educativas no México em matéria de ensino de arte e música, no contexto da Reforma Educacional de 2016, a qual em muitos sentidos foi controversa e não recebeu a acolhida esperada pelos agentes educacionais envolvidos no sistema educativo nacional e pelos cidadãos em geral. Ademais, em vista das recentes mudanças políticas, o próximo governo federal presidido por Andrés Manuel López Obrador, se posicionou por realizar uma consulta nacional para derrogação da mencionada reforma. Dada a incerteza atual em matéria educacional, esta análise apresente o panorama atual da educação artística, em pleno processo de transição política. A análise sugere que tanto a legislação como os planos e programas de estudo mostram consistência com o discurso político, orientado para o êxito de uma educação de qualidade, com suportes teóricos e fundamentos curriculares alinhados com as tendências internacionais em matéria de educação. Contudo, a operacionalização do novo modelo educativo, no que se refere à educação artística e musical, apresenta problemas e desafios substanciais para a sua adequada implementação, e, mais do que isso, enfrenta o desafio da sua própria permanência diante deste devir histórico. A análise dos programas de artes atuais revela um retrocesso no posicionamento da educação artística dentro do currículo, em comparação com os planos anteriores de estudo. Em que medida este posicionamento da educação artística melhora, em virtude da atual transição política, está ainda por ser verificado.

Palavras-chave: Artes e Educação. Reforma Educacional. Planos e Programas de Estudo. Educação Musical. Políticas Educacionais. 


\section{Introducción}

Uno de los temas de mayor controversia en las políticas públicas en México se relaciona con la reforma educativa, impulsada por el anterior gobierno federal, presidido por el presidente Enrique Peña Nieto, y aprobada por la Cámara de Diputados el 20 de diciembre de 2012 (Boletín 0767, 2012), publicada en el Diario Oficial de la Federación el 26 de febrero de 2013. El discurso político sostiene que la reforma ha buscado asegurar la calidad de la educación básica, aumentar la cobertura y la calidad de la educación media superior y la educación superior, y recuperar la rectoría del gobierno federal del sistema educativo nacional. Esto se da mediante el fomento a la equidad e inclusión educativa, el incremento al acceso de los servicios educativos y la participación activa de padres, docentes, directivos y sociedad en general. Sin embargo, esta reforma no ha estado exenta de críticas y movimientos docentes que cuestionan sus fundamentos en materia administrativa y curricular (DÍAZ BARRIGA, 2017a, 2017b; SINEMBARGO, 2013). Hay quienes afirman que no se trata de una reforma educativa, sino de naturaleza administrativa, para un mayor control del estado (NOTIMÉRICA, 2017). En este sentido se argumenta que la "reforma educativa" se reduce a la evaluación docente, para la cual se gasta más en evaluarlos que en formarlos (GIL ANTÓN, 2016). Por su parte, el presidente electo de México, Andrés Manuel López Obrador, desde su campaña política criticó reiteradamente la reforma educativa por ser un "mecanismo de sometimiento al magisterio nacional" (LÓPEZ OBRADOR, 2017, p. 1). En sus propias palabras, señalaba que "la reforma educativa ni siquiera es una idea original, es parte de las llamadas reformas estructurales impuestas desde el extranjero, esa reforma no funcionará” (p. 2).

En este artículo presento un análisis del posicionamiento de la educación artística y musical dentro de los planes de estudio de educación básica, tomando en cuenta la legislación educativa actual, en el marco de la aún vigente Reforma Educativa 2016. Ante los recientes cambios políticos, el actual gobierno federal, bajo el liderazgo del presidente electo Andrés Manuel López Obrador, se ha encaminado a realizar una consulta nacional para la derogación de dicha reforma. Sin embargo, dada la incertidumbre actual en materia educativa, este análisis presenta el panorama actual de la educación artística, en pleno proceso de transición política, reconociendo que a partir del $1.0^{\circ}$ de diciembre de 
2018 podrían iniciarse cambios estructurales en el sistema educativo mexicano, o, como ha sucedido en sexenios anteriores, que los programas y planes de estudio se vean mínimamente afectados y no exista un impacto significativo en materia curricular. Tomando esto en cuenta y para claridad del artículo, describo primero las leyes y normativas que rigen la educación en México al momento actual, y sin pretender especular sobre los futuros cambios al corto o mediano plazo, poniendo particular atención a aquellos elementos relacionados con el desarrollo cultural y artístico, para posteriormente discutir sobre los planteamientos curriculares de la Reforma Educativa 2016 y sus implicaciones en la práctica real de la enseñanza y aprendizaje artístico-musical en la educación básica.

\section{Políticas públicas en materia de educación, cultura y artes}

Una de las prioridades de la mayoría de los gobiernos del mundo es el mejoramiento del sector educativo. México no es la excepción, sus políticas públicas van orientadas a establecer metas y compromisos hacia la mejora en el campo educativo en sus diferentes niveles y contextos, alineadas además a las tendencias internacionales en materia de educación (MIRANDA, 2018). La Constitución Política de los Estados Unidos Mexicanos establece en su artículo 3. que "toda persona tiene derecho a recibir educación” y que, además, "la educación que imparta el Estado tenderá a desarrollar armónicamente, todas las facultades del ser humano" (CONGRESO DE LA UNIÓN, 1917/2016, p. 5). Para cumplir con esta obligación constitucional, la Secretaría de Educación Pública (SEP) es la instancia rectora del sistema educativo mexicano, el cual se ha caracterizado por su carácter altamente centralizado. A nivel nacional, la SEP se encarga de elaborar y poner en función sus planes y programas de estudio tanto de la educación básica, media superior y normal.

El Plan Nacional de Desarrollo en México (PND, GOBIERNO DE LA REPÚBLICA, 2013), en su diagnóstico de la educación en materia cultural, reconoció que las actividades culturales y artísticas que se realizan en el país carecen de la debida difusión y son apreciadas por un número reducido de ciudadanos. De acuerdo al PND, la tercera de las 
cinco metas nacionales era lograr una educación de calidad y establecía que la educación debe impulsar "el desarrollo de las capacidades y habilidades integrales de cada ciudadano, en los ámbitos intelectual, afectivo, artístico y deportivo, al tiempo que inculque los valores por los cuales se defiende la dignidad personal y la de los otros" ( $p$. 59). Por lo tanto, entre los objetivos específicos de dicha meta estaba el de "ampliar el acceso a la cultura como un medio para la formación integral de los ciudadanos" (Objetivo 3 de la Meta Nacional, PND, p. 67).

Alineado a esta meta nacional, en particular, el Programa Sectorial de Educación (PSE 2013-2018) estableció como objetivo "promover y difundir el arte y la cultura como recursos formativos privilegiados para impulsar la educación integral” (Objetivo 5 del PSE, SEP, 2013, p. 38). De particular importancia, el PSE reconocía que:

La cultura es un componente esencial para alcanzar una educación integral, una herramienta para el desarrollo, eje fundamental para la articulación social y garante de la paz. Difundir las manifestaciones culturales promueve el conocimiento y aceptación de la diversidad social y cultural, la tolerancia. La cultura debe situarse en un lugar sobresaliente en el proyecto educativo nacional y contribuir a la formación integral de niñas, niños y jóvenes propiciando un mayor acceso a los bienes y servicios del arte y la cultura. (PSE, p. 63)

Para el logro de este objetivo de difusión del arte y la cultura orientado a impulsar la educación integral de la población, el PSE establecía cinco estrategias, con sus respectivas líneas de acción (SEP, 2013, pp. 64-65, ver Tabla 1).

\section{Tabla 1.}

Estrategias y líneas de acción en materia de educación artística y cultural del Programa Sectorial de Educación (SEP, 2013, pp. 64-65).

\begin{tabular}{l} 
Estrategia 5.1. Fomentar la educación artística y cultural y crear mayores oportunidades \\
de acceso a la cultura, especialmente para el sector educativo \\
\hline Líneas de acción \\
5.1.1. Ampliar las opciones de iniciación y apreciación artística para fortalecer la \\
formación integral en la educación básica y media superior \\
5.1.2. Elaborar alternativas de capacitación para que el personal docente de educación \\
básica y media superior desarrolle contenidos artísticos y culturales. \\
5.1.3. Fomentar la lectura como habilidad básica en la superación de la desigualdad. \\
\hline
\end{tabular}


5.1.4. Fortalecer la educación media superior y la educación superior en materia artística y cultural.

5.1.5. Otorgar becas e incentivos para apoyar la educación artística y cultural.

5.1.6. Propiciar el acceso de estudiantes y docentes a actividades artísticas y culturales de nivel internacional para fortalecer la apreciación artística.

5.1.7. Eliminar barreras que impiden el acceso de población con discapacidad a las actividades artísticas y culturales.

Estrategia 5.2. Dotar a la infraestructura cultural de espacios y servicios dignos

Líneas de acción

5.2.1. Desarrollar acciones de recuperación, rehabilitación, mantenimiento y equipamiento de la infraestructura cultural en apoyo al proceso educativo.

5.2.2. Desarrollar nuevos espacios para actividades culturales, multifuncionales y comunitarios, en zonas urbanas marginadas.

5.2.3. Prever que las acciones de infraestructura cultural atiendan los requisitos de las personas con discapacidad.

Estrategia 5.3. Fortalecer la identidad nacional a través de la difusión del patrimonio cultural y el conocimiento de la diversidad cultural

Líneas de acción

5.3.1. Incrementar las acciones para un mayor conocimiento y difusión del patrimonio cultural, en especial para estudiantes y docentes.

5.3.2. Difundir las expresiones de las culturas populares, indígenas, urbanas y comunitarias, destacando su dinamismo y capacidad de innovación e interacción.

5.3.3. Considerar las aportaciones de las culturas locales como elementos enriquecedores de la educación.

Estrategia 5.4. Reforzar los canales de generación y acceso a bienes y servicios culturales para la población estudiantil

Líneas de acción

5.4.1. Desarrollar nuevos contenidos para niños y jóvenes en la radio y televisión culturales.

5.4.2. Fomentar la producción de libros mediante coediciones y tecnologías digitales.

Estrategia 5.5. Fortalecer el acceso de la población estudiantil y docente a la cultura, con el uso de las tecnologías digitales

Líneas de acción

5.5.1. Generar contenidos culturales en formatos digitales multimedia para apoyo de los programas educativos.

5.5.2. Publicitar, a través de plataformas tecnológicas, las actividades culturales y artísticas dirigidas a los estudiantes y docentes.

5.5.3. Crear plataformas y servicios digitales que favorezcan una oferta amplia de contenidos culturales, especialmente para niñas, niños y jóvenes.

5.5.4. Estimular la creación de proyectos vinculados a la ciencia, la tecnología y el arte, que ofrezcan contenidos para plataformas digitales.

Para la consecución de la meta nacional, el PSE 2013-2018 reconocía que es fundamental la vinculación entre el sector educativo y el sector cultural, particularmente 
en los niveles de educación básica y media superior. Es por esto que, en concordancia con las metas nacionales del PND y los objetivos del PSE, se elaboró el Programa Especial de Cultura y Arte (PECA) 2014-2018, a ejecutarse por el entonces Consejo Nacional para la Cultura y las Artes (Conaculta), y actualmente por la Secretaría de Cultura (creada en diciembre de 2015), rectora de las políticas públicas en materia cultural y artística. EI PECA establecía que:

Deberá reconocerse, ineluctablemente, el concepto de transversalidad de la tarea cultural como una estrategia de planeación y colaboración y la necesidad de impulsar una política cultural de carácter nacional bajo esquemas de colaboración con estados y municipios, y de participación con las comunidades artística e intelectual, con organismos privados y académicos y, fundamentalmente, con la sociedad. (SEP, 2014b, p. 5)

El objetivo 2 del PECA hace alusión directa a la necesidad de impulsar la educación y la investigación artística y cultural (SEP, 2014, p. 9), fortaleciendo la labor de los centros de investigación y ampliando el trabajo interdisciplinar. Se enfatizaba también el rol de la Secretaría de Cultura y sus organismos coordinados en la elaboración de contenidos artísticos y culturales digitales, orientados a la educación básica y media superior para el desarrollo integral de niños y jóvenes. Establecía la necesidad de revisar y actualizar los programas educativos en relación con las artes y la cultura, con la finalidad de lograr altos estándares en la formación de los estudiantes. Dentro de este objetivo, además, se estipulaba la meta de involucrar a estudiantes, docentes y a la comunidad educativa en general en las diversas manifestaciones artísticas. Las estrategias y líneas de acción que se proponían para lograr este objetivo se presentan a continuación (Tabla 2).

\section{Tabla 2}

Estrategia y líneas de acción para impulsar la educación e investigación artística del Programa Especial de Cultura y Arte (SEP, 2014, p. 16).

Estrategia 2.1. Enriquecer la educación en materia artística y cultural
Líneas de acción
2.1.1. Desarrollar, impulsar y fortalecer los programas de educación en materia artística
y cultural.
2.1.2. Fortalecer la educación artística y cultural a través de programas de extensión
académica.
2.1.3. Ampliar y enriquecer la oferta académica de las artes mediante la educación a


distancia.

2.1.4. Otorgar becas e incentivos para apoyar la educación artística y cultural. 2.1.5. Vincular a las escuelas de artes escénicas con espacios de representación profesional.

2.1.6. Elaborar alternativas de capacitación para que el personal docente desarrolle contenidos artísticos y culturales.

Algunos de los programas que prevalecen y han vinculado la cultura con la educación, tanto formal como no formal y de carácter comunitario, incluyen el programa Alas y Raíces, que privilegia la participación de niños y jóvenes como generadores de cultura, desarrollando su creatividad y pensamiento crítico en proyectos de producción artística (COORDINACIÓN NACIONAL DE DESARROLLO CULTURAL INFANTIL, 2013). Otros programas orientados a la educación artística con carácter social y comunitario es el denominado Cultura para la Armonía (SECRETARíA DE CULTURA, 2017), el cual tiene como objetivo posicionar las diferentes manifestaciones artísticas y prácticas culturales locales como herramientas para favorecer la cohesión social y el desarrollo humano. El programa se rige por tres principios rectores: (a) tiempo creativo, para la recuperación del espacio público en zonas de alta vulnerabilidad o riesgo; (b) arte en armonía, integrando a niños y jóvenes en agrupaciones artísticas comunitarias que promueven el trabajo en equipo, la convivencia y la colaboración; y (c) culturas vivas, reconociendo y preservando las buenas prácticas del patrimonio cultural regional.

\section{Consulta sobre la reforma y el modelo educativo 2016 en relación a las artes}

En septiembre de 2013, la Secretaría de Educación Pública abrió una convocatoria a la ciudadanía y todos aquellos actores involucrados en la educación básica, media superior y normal del país, para que se presentaran propuestas y participaran en los foros de consulta nacional para la revisión del modelo educativo planteado por la SEP (2014a). Esta Consulta nacional se realizó de febrero a junio de 2014. Posteriormente, el 20 de julio de 2016, la SEP dio a conocer tres documentos que conformaron la propuesta educativa: (1) Los Fines de la Educación en el Siglo XXI (SEP, 2016a), que describía de manera breve el tipo de ciudadano que se buscaba formar mediante el modelo educativo propuesto; (2) el 
Modelo Educativo 2016, que describía el planteamiento pedagógico de la reforma educativa (SEP, 2016b); y (3) la Propuesta Curricular para la Educación Obligatoria (SEP, 2016c), que presentaba en detalle la organización de los aprendizajes para la educación básica y media superior. A partir de esa misma fecha y hasta el 30 de septiembre de 2016, la SEP convocó nuevamente a una consulta nacional pública con el objetivo de conocer la opinión de la ciudadanía respecto a estos tres documentos.

En materia de educación artística, la consulta sobre el Modelo Educativo 2017 (CIDE, 2016) resaltó que la ciudadanía valora en gran medida la inclusión de actividades artísticas como estrategia de acercamiento a la cultura, en la construcción de identidad y como forma de expresión. Sin embargo, el reporte confirmó además que existe una percepción generalizada respecto a la falta de recursos e infraestructura para llevar a cabo las actividades artísticas. Como en anteriores programas nacionales de educación, se percibe que las artes no son consideradas tan importantes como otras asignaturas, al no ser incluidas dentro de los aprendizajes clave. Además, el tiempo asignado en la primaria es considerado insuficiente, más cuando el planteamiento curricular concentra todas las artes en una misma asignatura, como si pudieran cubrirse juntas. Como se hace explícito en el reporte:

Preocupa que en la Propuesta Curricular no se desarrolla el cómo, ni el perfil de quién podría hacerse cargo del desarrollo artístico. De igual forma, inquieta que se haga énfasis en "que se aprecie la belleza", pues el arte conlleva muchas otras implicaciones. Plantearlo de esa forma, les parece ambiguo y limitante. Además, se critica el incluir a todas las artes en un "mismo paquete", sobre todo cuando, en la dosificación curricular, únicamente se le destinará una hora a la semana. Se considera indispensable aumentar las horas destinadas al arte en las escuelas. Debe tomarse en cuenta que los alumnos son capaces de desarrollar un pensamiento artístico crítico, y que el arte es mucho más integral de lo que se plantea en la propuesta. En el mismo sentido, preocupa el perfil del docente. Señalan que un buen docente para este desarrollo, tendría que saber música, danza, pintura, teatro. (CIDE, 2016, p. 49)

Entre las recomendaciones más importantes en materia de educación artística surgidas de esta consulta nacional se incluyeron el considerar el trabajo artístico como un aprendizaje clave para lograr una educación integral; considerar el arte como una 
experiencia creadora de conocimiento, no solo enfocado en la ejecución o apreciación artística; la necesidad de revisar las secuencias curriculares; enfatizar la práctica transdisciplinaria para el desarrollo artístico; en el área de música, al considerarse una práctica colectiva, que se incorporaran ensambles y colectivos artísticos; y, de particular importancia, se recomendó que el arte se dividiera en cada especialidad artística.

Después de realizada la segunda consulta nacional, en 2017 se presentaron los tres documentos de la reforma educativa en su versión final. A continuación se describen sus lineamientos en materia de educación artística y más específicamente en educación musical, los cuales, como podrá observarse, atendieron mínimamente las recomendaciones surgidas de la consulta nacional.

\section{Los fines de la educación en el siglo XXI}

El primero de los documentos de la Reforma Educativa 2016 es la Carta denominada Los Fines de la Educación en el Siglo XXI (SEP, 2017a, 2017c), la cual establece que todo egresado de educación básica y media superior debe ser una persona que, entre otras habilidades ahí descritas, "tenga creatividad y un sentido estético, aprecie la cultura y las artes" (SEP, 2017a, p. 2). El documento describe los logros esperados al término de cada nivel educativo en cada área del currículo. La Tabla 3 muestra las metas específicas en la asignatura de Apreciación y Expresión Artísticas.

\section{Tabla 3}

Aprendizajes esperados para el área de Apreciación y Expresión Artísticas (SEP, 2017, p. 4).

\begin{tabular}{|c|c|c|c|c|}
\hline Ámbito & $\begin{array}{l}\text { Al término del } \\
\text { preescolar }\end{array}$ & $\begin{array}{l}\text { Al término de la } \\
\text { primaria }\end{array}$ & $\begin{array}{l}\text { Al término de la } \\
\text { secundaria }\end{array}$ & $\begin{array}{ll}\text { Al término de la } \\
\text { educación } \\
\text { superior }\end{array}$ \\
\hline $\begin{array}{l}\text { Apreciación y } \\
\text { Expresión } \\
\text { Artísticas }\end{array}$ & $\begin{array}{lr}\text { Desarrolla } & \text { su } \\
\text { creatividad } & \text { e } \\
\text { imaginación } & \text { al } \\
\text { expresarse con } & \text { recursos de las artes } \\
\text { (por ejemplo, las } \\
\text { artes visuales, la } \\
\text { danza, la música y el } \\
\text { teatro) }\end{array}$ & $\begin{array}{l}\text { Explora y experimenta } \\
\text { distintas } \\
\text { manifestaciones } \\
\text { artísticas. Se expresa } \\
\text { de manera creativa } \\
\text { por medio de } \\
\text { elementos de la } \\
\text { música, la danza, el } \\
\text { teatro y las artes } \\
\text { visuales }\end{array}$ & $\begin{array}{l}\text { Analiza, aprecia y } \\
\text { realiza distintas } \\
\text { manifestaciones } \\
\text { artísticas. Identifica y } \\
\text { ejerce sus derechos } \\
\text { culturales } \\
\text { ejemplo, el derecho a } \\
\text { practicar } \\
\text { costumbres sus } \\
\text { tradiciones). Aplica } \\
\text { su creatividad para }\end{array}$ & $\begin{array}{l}\text { Valora y experimenta } \\
\text { las artes porque le } \\
\text { permiten comunicarse, } \\
\text { le aportan un sentido } \\
\text { de identidad y } \\
\text { comprende al } \\
\text { contribución a } \\
\text { desarrollo integral de } \\
\text { las personas. Aprecia } \\
\text { la diversidad de las } \\
\text { expresiones culturales. }\end{array}$ \\
\hline
\end{tabular}




\begin{tabular}{|l|l|l|}
\hline & & $\begin{array}{l}\text { expresarse por } \\
\text { medio de elementos } \\
\text { de las artes (entre } \\
\text { ellas, la música, la } \\
\text { danza y el teatro) }\end{array}$ \\
\hline
\end{tabular}

\section{Modelo educativo 2016}

En el documento del Modelo Educativo para la Educación Obligatoria (SEP, 2017b) se argumenta la necesidad de un replanteamiento del sistema educativo nacional en su conjunto, desde los planes y programas, los materiales y los métodos educativos. El Modelo se basa en cinco grandes ejes que reorganizan el sistema educativo, y que buscan desarrollar el potencial de niñas, niños y jóvenes.

El primer eje es el Planteamiento Curricular, con un enfoque humanista concentrado en el desarrollo de aprendizajes clave. En el resumen ejecutivo se dice que estos "contribuyen al desarrollo integral de los estudiantes que les permiten aprender a lo largo de la vida [...] Adicional a los campos de la formación académica, se incorpora el desarrollo personal y social de los estudiantes como parte integral del currículo, con énfasis especial en el desarrollo de las habilidades emocionales" (SEP, 2016b, p. 27). Es importante señalar que, a diferencia del plan anterior, se otorga a las escuelas cierto nivel de autonomía curricular al ofrecer la posibilidad de incorporar otras asignaturas o contenidos de relevancia para el contexto educativo específico.

El segundo eje coloca La Escuela al Centro del Sistema Educativo como unidad básica de organización del sistema educativo, con autonomía de gestión y presupuesto propio, mayor liderazgo directivo y trabajo colegiado, pero menor carga administrativa y burocrática.

El tercer eje lo constituye la Formación y Desarrollo Profesional Docente. Se establece el Servicio Profesional Docente, un sistema basado en el mérito y "que define los mecanismos para el ingreso, la promoción, el reconocimiento y la permanencia de los maestros" (SEP, 2016b, p. 54). 
En el cuarto eje Inclusión y Equidad, se establecen las bases para que todos los estudiantes, independientemente de su origen étnico, lengua materna, género, condición socioeconómica, aptitudes sobresalientes o discapacidad, cuenten con las mismas oportunidades para el apropiado desarrollo de sus potencialidades.

El último eje sobre la Gobernanza del Sistema Educativo se relaciona con aquellos mecanismos institucionales que faciliten una efectiva operación administrativa en el sistema educativo mexicano, la colaboración entre ámbitos de gobierno, el papel del Instituto Nacional para la Evaluación de la Educación, la relación entre la autoridad educativa y el magisterio, la participación de las familias en la educación, así como la relación entre organizaciones de la sociedad civil, academia y sector productivo.

\section{Las artes en la educación obligatoria (Educación Básica y Media Superior)}

De acuerdo al nuevo modelo educativo, el mapa curricular de la educación obligatoria está conformado por "tres componentes curriculares: Campos de Formación Académica; Áreas de Desarrollo Personal y Social; y Ámbitos de la Autonomía Curricular, a los que, en conjunto, se denomina Aprendizajes Clave para la Educación Integral" (SEP, 2017c, pp. 107-108). El primero, denominado Campos Formativos de la Educación Básica incluye tres áreas: (a) Lenguaje y comunicación (con asignaturas como: español, inglés, lengua materna, literatura, dependiendo del nivel educativo); (b) Pensamiento matemático (matemáticas, álgebra, aritmética, cálculo, trigonometría); y (c) Exploración del mundo natural y social (ciencias naturales, ciencias sociales, geografía, formación cívica y ética, historia). Antes de la consulta nacional, únicamente estas tres áreas correspondían a los aprendizajes clave, dejando fuera de esta denominación al resto de las asignaturas. El segundo componente denominado Áreas de Desarrollo Personal y Social está integrado por las áreas de: (a) Artes, que en educación básica (preescolar a secundaria) incluye las cuatro disciplinas artísticas de Música, Artes Visuales, Teatro y Danza, mientras que en educación media superior (nivel preparatoria) está constituida por materias como Estética y Literatura, así como por otras asignaturas humanísticas como Filosofía, Ética y Lógica; además incluye a las áreas de (b) Educación Física y (c) 
Educación socioemocional y tutoría. El tercer componente lo incluyen ámbitos de aprendizaje que son directamente definidos por la escuela, con base en lineamientos de la propia SEP, entre los que se podrían considerar la profundización de los aprendizajes clave, la ampliación de actividades enfocadas al desarrollo personal y social, los nuevos contenidos de relevancia en su formación, el conocimiento de contenidos regionales y locales, y los proyectos de impacto social.

Como puede observarse en el nuevo modelo educativo, la educación del arte sigue formando parte de las enseñanzas en nivel básico, desde preescolar hasta la secundaria. Para el primer y el segundo grado de nivel preescolar, las artes tienen asignadas un total de 2 horas a la semana (80 horas anuales) tanto en escuelas con jornada regular como de tiempo completo, representando el $15 \%$ y el 5,6\% del tiempo escolar. Sin embargo, en el tercer grado de preescolar, el tiempo asignado a las artes se ve reducido a 1,5 horas a la semana (60 horas anuales). Para la educación primaria (SEP, 2017C), las artes tienen asignadas únicamente una hora a la semana (40 horas al año), incluso en las escuelas de tiempo completo, a las que el plan de estudios anterior asignaba dos horas (SEP, 2011), lo que muestra un detrimento a la posición de las artes en este nivel educativo. Mientras que en la jornada regular la educación del arte representa el 4,4\% del tiempo escolar, en las escuelas de tiempo completo solo cubre el 2,5\% del tiempo en todos los grados de la educación primaria. Es importante resaltar que en esa hora asignada semanalmente al área de artes se deben abordar los contenidos de las cuatro disciplinas artísticas, lo que implicaría un cuarto de hora para la educación musical, si la división del tiempo fuera equitativa entre las disciplinas. En la educación secundaria, las artes tienen asignadas tres horas en jornada regular, lo que representa el 8,5\% del tiempo escolar, mientras que las mismas horas son asignadas en escuelas de tiempo completo, cubriendo solo el $6,7 \%$ del tiempo.

La educación artística en la educación básica (preescolar-secundaria) se basa en un concepto amplio de las artes enfocado en el reconocimiento de la diversidad cultural y artística de México y el mundo, y que se plantea como incluyente al dar cabida no solo las llamadas bellas artes, sino también las artes populares, indígenas, tradicionales, contemporáneas y emergentes (SEP, 2017c). El enfoque pedagógico se centra en dos 
componentes curriculares clave: la expresión artística (procesos creativos y libertad de expresión) y la apreciación artística. A continuación se presentan las características básicas de la educación artística en los tres niveles educativos de la educación básica.

Artes en la educación preescolar. En este nivel educativo, la asignatura de Artes tiene dos objetivos centrales, el que permitan al estudiante: (1) "usar la imaginación y la fantasía, la iniciativa y la creatividad para expresarse por medio de los lenguajes artísticos”; e (2) "identificar manifestaciones artísticas y culturales de su entorno y de otros contextos" (p. 467).

Artes en la educación primaria. Los objetivos de la educación artística en la escuela primaria es desarrollar en los estudiantes habilidades para:

(1) Explorar los elementos básicos de las artes desde una perspectiva interdisciplinaria.

(2) Experimentar con las posibilidades expresivas de los elementos básicos de las artes.

(3) Promover el desarrollo del pensamiento artístico al explorar procesos de percepción, sensorialidad, emoción, imaginación, creatividad y comunicación.

(4) Reconocer las artes como manifestaciones culturales de la sociedad o grupo donde se producen, valorando la variedad y diversidad de expresiones.

(5) Identificar las etapas en la realización de un proyecto artístico.

(6) Desarrollar las capacidades emocionales e intelectuales para apreciar las manifestaciones artísticas.

(7) Propiciar ambientes de aprendizaje que permitan el intercambio y la comunicación abierta y respetuosa acerca del arte.

(8) Favorecer actitudes de respeto, apertura al cambio y manejo de la incertidumbre, imaginando y proponiendo soluciones creativas a diversas problemáticas que se presenten en el colectivo artístico interdisciplinario. (SEP, 2017c, p. 469)

En la primaria se propone un modelo integrador el cual se conjugan los contenidos de las disciplinas artísticas en un "colectivo artístico interdisciplinario" (p. 473). Sin embargo, el documento curricular sugiere que en el primer y el segundo grados de la primaria se trabaje con música y danza, en el tercer y el cuarto se aborden las artes 
visuales, y en el quinto y el sexto grados se enfoque en teatro (p. 474). Aunque esta es solo una sugerencia que pudo haber surgido de la petición de que el arte se dividiera en cada especialidad artística, como se reportó en la consulta nacional, esto implicaría concentrar los contenidos curriculares de las disciplinas artísticas a solo dos años, descuidándolas en los cuatro años restantes de la educación primaria.

Artes en la educación secundaria. De acuerdo al plan de estudios (SEP, 2017C), los propósitos de la educación artística en la educación secundaria son:

(1) Explorar los elementos básicos del arte en una de las disciplinas artísticas (artes visuales, danza, música o teatro), y utilizarlos para comunicarse y expresarse desde una perspectiva estética.

(2) Consolidar un pensamiento artístico al profundizar en los aspectos de percepción, sensorialidad, imaginación, creatividad y comunicación, reconociendo las conexiones entre ellos.

(3) Valorar las manifestaciones artísticas en su dimensión estética al utilizar sus capacidades emocionales e intelectuales.

(4) Analizar las etapas en la realización de proyectos artísticos a partir de la investigación e indagación de propuestas locales, nacionales o internacionales de artes visuales, danza, música o teatro.

(5) Explorar las artes visuales, danza, música o teatro desde un enfoque sociocultural que les permita reconocer su importancia en la sociedad y ejercer sus derechos culturales.

(6) Fortalecer actitudes de respeto a la diversidad, apertura al cambio y manejo de la incertidumbre en su actuación cotidiana, a partir del trabajo con las artes visuales, danza, música o teatro. (SEP, 2017c, p. 470)

En la secundaria se sugiere el trabajo por proyectos artísticos, individuales o colectivos en una sola disciplina de elección para el estudiante. Se espera que los estudiantes cursen la misma disciplina artística durante los tres grados de la secundaria. Este planteamiento curricular tiene implicaciones directas respecto al qué, cómo y quién debe impartir la enseñanza de las artes en los diferentes niveles educativos. Se sugiere que en primaria sea el maestro normalista el encargado de impartir la asignatura de Artes 
y en secundaria sean maestros especialistas en alguna de las cuatro disciplinas artísticas. Se deja a discreción de las escuelas secundarias las disciplinas artísticas que están en posibilidades de ofrecer de acuerdo al perfil de sus docentes. Es importante resaltar que el perfil deseable del docente es ser "un generador de situaciones de aprendizaje mediante el método de proyectos y el fortalecimiento de experiencias educativas, y que considere a los estudiantes como sujetos activos capaces de producir sus propios aprendizajes" (p. 475).

\section{Enfoque pedagógico de la educación musical en Educación Básica}

En lo que respecta a la educación musical, específicamente el enfoque pedagógico, se centra en dar oportunidades a los niños y jóvenes para participar en procesos de creación y expresión musicales, donde se estimulen su sensibilidad, iniciativa, creatividad y gusto estético. Se hace especial énfasis en que las diversas experiencias de expresión y apreciación musicales estén relacionadas con la cultura a la que pertenecen los estudiantes y que también se provea el acceso al conocimiento de manifestaciones musicales de otras partes del país y del mundo. Al final de la educación básica, se espera que los estudiantes valoren el rol e importancia de las diferentes manifestaciones musicales locales, nacionales e internacionales, como resultado de la exploración y experimentación sistemática y gradual de los elementos musicales (SEP, 2017C).

Tanto en nivel preescolar como en nivel primaria se hace gran énfasis en el juego como enfoque didáctico (SEP, 2017C). Con las actividades musicales se busca brindar oportunidades para que los niños escuchen música y respondan físicamente al ritmo, esto como estrategia para desarrollar la atención y la receptividad, tanto en actividades de creación como en las que implican la audición y apreciación musicales. Se deben propiciar actividades para el reconocimiento y discriminación de sonidos, desarrollo de la memoria musical, así como la formación de criterios de selección, de acuerdo con gustos y preferencias (SEP, 2017c, p. 471).

En la primaria se da continuidad a las actividades de expresión y apreciación musical y se avanza hacia la realización de un colectivo artístico interdisciplinario (SEP, 
2017c, p. 473), donde en cada ciclo escolar se exploran opciones temáticas que permitan relacionar las diferentes artes, eligiendo canciones, técnicas, textos teatrales, entre otros elementos, para generar una obra artística que se presentará ante un público. Es importante resaltar nuevamente que la asignación horaria en este nivel es de una hora a la semana, y que se recomienda centrar esfuerzos en el área musical únicamente en el primer y segundo grados.

En la secundaria, el enfoque pedagógico es por medio de proyectos artísticos más amplios en nivel secundaria y se recomienda considerar los intereses y preferencias de los jóvenes, "buscando impulsar la exploración consciente, la libertad de expresión y el pensamiento crítico" (SEP, 2017c, p. 474). Se busca que los estudiantes conformen un grupo artístico vocal donde todos participen en el montaje de un repertorio, y se trabaje organizadamente en ensayos y presentaciones públicas. El elemento básico es el canto y complementariamente se contempla el uso de instrumentos (i.e., el cuerpo como instrumento de percusión, instrumentos de percusión tradicionales construidos con material reciclable, o instrumentos que sepan tocar los mismos estudiantes) o elementos escénicos (movimientos escénicos, vestuario, escenografía, etc.) (p. 504). La carga horaria es de tres horas y se espera que las instituciones cuenten con profesores especializados en alguna de las cuatro áreas artísticas.

Lo que llama la atención es la transversalidad de los contenidos y como ahora, explícitamente, se incluyen actividades musicales que favorecen el aprendizaje de otras asignaturas. Como se manifiesta en el plan de estudios, "Ios contenidos de los programas de Artes en educación básica promueven la relación con otros campos de Formación Académica y Áreas de Desarrollo Personal y Social desde una perspectiva interdisciplinaria, lo que permite transferir sus estructuras de conocimiento a otras asignaturas y áreas" (SEP, 2017c, p. 468). Por ejemplo, en los seis grados de la educación primaria, así como en los tres grados de la educación secundaria, el programa de estudios establece la "lectura y escucha de poemas y canciones" como una de las prácticas sociales del lenguaje que debe ser sistemáticamente aplicada. Como aprendizajes esperados, el estudiante deberá ser capaz de cantar, leer y reescribir canciones y rondas infantiles, pero además debe ser capaz de aprender y reinventar rondas infantiles, 
creando y jugando con el juego poético (SEP, 2017C, p. 190). Con este tipo de actividades se trata de fortalecer no solo el lenguaje, sino también el sentido de identidad (quién habla, a quién se dirige, para qué), se busca que sea capaz de interpretar los mensajes, así como identificar características de los géneros musicales, y elementos del lenguaje como estrofas, temas, versos, entre otros. De igual forma se establece el uso de otros recursos artísticos como la "lectura, escritura y escenificación de obras teatrales" como otra de las prácticas sociales para el desarrollo del lenguaje en los seis grados de la educación primaria.

Hasta aquí, se ha buscado presentar el panorama general de las políticas públicas y cómo estas determinan el posicionamiento actual de la educación artístico-musical dentro del currículo. A continuación se discuten las implicaciones que tienen las políticas públicas en la educación artístico-musical, cómo difieren de la realidad educativa y los retos que aún se tienen que enfrentar, considerando el marco actual de la Reforma Educativa 2016.

\section{Discusión}

Al igual que en muchos otros países del mundo, la legislación educativa actual mexicana, independientemente del momento particular de transición política en este país, va encaminada a establecer las bases y principios para el logro de una educación de calidad, equitativa e incluyente. Mientras que el discurso político educativo es consistente con las tendencias internacionales (MIRANDA, 2018) es importante examinar la brecha existente entre las políticas y la realidad educativa. Claramente, los documentos oficiales enfatizan la importancia de la cultura y las artes dentro de una educación integral y el Estado se responsabiliza de la provisión de oportunidades para que los estudiantes accedan a experiencias artísticas y a su formación en sus diversas disciplinas. Sin embargo, se reconoce que en materia cultural las artes aún carecen de la debida difusión, a pesar del reconocimiento que se da al impacto que tienen en los ámbitos intelectual y afectivo (PND, 2013).

Mientras que los diversos programas educativos y culturales establecen metas, estrategias y líneas de acción, los indicadores para medir el logro de los objetivos tienden 
a ser simplistas o reduccionistas. Los pocos indicadores que están plenamente descritos hacen referencia al porcentaje de participación de la población nacional en actividades artísticas y culturales, los índices de eficiencia terminal en escuelas de educación media superior y de educación superior enfocadas específicamente a la formación artística, la proporción de alumnos beneficiados con becas o cursos de capacitación dentro del sistema escolarizado de educación artística, el número de acciones de capacitación para promotores culturales o el número de estudiantes que asisten a servicios culturales como bibliotecas, museos, salas de conciertos (GOBIERNO DE LA REPÚBLICA, 2017). En materia de educación artística y musical, en el sistema de educación básica obligatoria no se han establecido indicadores precisos que permitan evaluar el avance en acceso y calidad en los diversos niveles educativos y subsistemas (estatal y federal, público y privado). Por ejemplo, las diferencias de la provisión de la educación artística y musical entre escuelas públicas y privadas es una muestra de cómo esta es un área en la que justamente se adolece de equidad. La inmensa mayoría de los estudiantes en el país, inscritos en escuelas públicas, no tienen acceso a una educación artística y musical de calidad. A diferencia de algunos estados del país que muestran un mayor compromiso hacia esta área de formación y favorecen la contratación de maestros especialistas en Artes (e.g., el Sistema Estatal de Educación del Estado de Chihuahua), muchos estados dependen en gran medida de la capacidad de los maestros normalistas para impartir los contenidos curriculares de Artes y Música. Aunque estos últimos reciben formación en educación artística, esta no necesariamente les permite enseñar las artes con la suficiente amplitud y profundidad. A esto hay que agregarle las limitadas oportunidades que tienen tanto maestros normalistas como especialistas de participar en programas de desarrollo profesional que eleve la calidad de su práctica docente (DIRECCIÓN GENERAL DE FORMACIÓN CONTINUA, ACTUALIZACIÓN Y DESARROLLO PROFESIONAL DE MAESTROS DE EDUCACIÓN BÁSICA, s.f.; GONZÁLEZ-MORENO, 2010). Es precisamente la formación y el desarrollo profesional docente uno de los temas de mayor debate en la reforma educativa (CIDE, 2016), por lo que se requieren procesos de capacitación continua que sean pertinentes y de calidad, no únicamente en los ámbitos de los aprendizajes clave de Español y Matemáticas, sino también en las Artes y en la Música en particular. 
Aunque el Programa Sectorial de Educación estableció como línea de acción la elaboración de programas de capacitación al personal docente, en el que se desarrollen contenidos artísticos y culturales, esto no se ha implementado aún; los docentes siguen dependiendo de las alternativas de capacitación que ofrecen otras instancias educativas o asociaciones no gubernamentales más cercanas a los intereses y necesidades individuales de los docentes de artes, en particular cuando los programas de capacitación continua ofrecidas por el estado se centran prioritariamente en las asignaturas de Español y Matemáticas. Entre las opciones externas que los maestros de Artes pueden considerar para elevar su nivel de profesionalización están los programas de pregrado y posgrado, o por medio de su participación en eventos académicos y talleres organizados por asociaciones no gubernamentales (e.g. ONGs de educación musical tales como ISME, FLADEM, FORMEDEN, entre otras), sin embargo, con limitado o nulo apoyo por parte de las autoridades educativas para cubrir los costos económicos o de tiempo requerido para este tipo de actividades. Aunque existe la opción de "beca comisión" en la que se autorizan permisos con goce de sueldo, estas son autorizadas de forma limitada y discrecional (SNTE, 2018).

El Programa Sectorial de Educación (2013) establecía como línea de acción (5.1.1) “ampliar las opciones de iniciación y apreciación artística para fortalecer la formación integral en la educación básica y media superior"; sin embargo, los programas de estudio para la educación básica (SEP, 2017c) muestran una marcada desigualdad en la inclusión de la educación musical en los diferentes niveles educativos. Mientras que el programa de estudio en nivel preescolar establece actividades de educación artística y musical en sus tres grados, los programas de la primaria se restringen a ofrecer contenidos de educación musical únicamente en el primer y segundo grados. Esta es una diferencia sustancial en comparación con los anteriores programas del estudio (SEP, 2011), donde sí se incluían contenidos de educación musical durante los seis grados de la primaria. Similar al plan curricular 2011, en la educación secundaria se da la opción de especializarse en una sola disciplina artística en particular, dependiendo de las áreas de especialización artística de los docentes que laboren en las instituciones educativas. En el caso de que una escuela cuente con la presencia de un maestro de Música, los alumnos interesados en esta 
disciplina podrían cursarla durante los tres años de su educación secundaria. La clase de Música se vuelve electiva y los estudiantes tienen limitadas oportunidades para desarrollar competencias en otras disciplinas artísticas, que podrían contribuir a una mayor comprensión de la interrelación entre las artes. Muchos otros estudiantes podrían simplemente no tener acceso a la educación musical en este nivel educativo. En nivel medio superior, se deja el modelo de las cuatro disciplinas artísticas básicas -Música, Artes visuales, Teatro y Danza - para incluir otras como Estética y Literatura. Este cambio deja fuera la posibilidad de que los estudiantes sigan desarrollando competencias musicales, excepto si existen opciones extracurriculares en las instituciones educativas.

En la consulta nacional sobre el modelo educativo (CIDE, 2016) se criticó el limitado referencial teórico en materia de la educación musical y del arte en general. Aunque los documentos revisados ya hacen referencia directa a concepciones teóricas de diversos autores como John Dewey, Lev Vigotsky, Elliot Eisner y Howard Gardner, entre otros (SEP, 2017c, p. 473), la aplicación práctica de dichas concepciones está vagamente descrita. En relación con los contenidos curriculares en Arte y Música, es evidente que pueden surgir dos puntos de vista contrastantes. Por un lado, hay quienes consideran que dichos contenidos curriculares son relativamente básicos y que la integración de los mismos con otras áreas curriculares puede verse facilitada por medio del trabajo integrador de los maestros normalistas (esto en nivel primaria), a pesar de las limitaciones en la formación de los docentes y en los limitados recursos didácticos y de infraestructura. Por otro lado, dado que los contenidos curriculares están pensados para que cualquier maestro, normalista o especialista pueda abordarlos, no es posible potenciar el desarrollo artístico-musical de los estudiantes, ya que se limitan a desarrollar competencias genéricas en las artes, sin llegar a una mayor profundización o conocimiento teórico-práctico de las artes y de la música en particular. Esto lleva a cuestionarse la viabilidad práctica del planteamiento curricular en este ámbito.

Lo rescatable del modelo educativo es la denominada "autonomía curricular", con la cual es factible flexibilizar e incluso expandir el currículo, incentivando la innovación y creatividad docente para acercar los contenidos a los intereses y habilidades de los estudiantes (Acuerdo n. ${ }^{\circ}$ 11/05/18, SEP 2018). Por medio de los denominados “clubes" se 
busca proveer oportunidades para que los estudiantes amplíen sus conocimientos, habilidades y experiencias con las artes. Se contempla que los clubes integren a estudiantes de diferentes grados escolares, en un horario escolar determinado. Los clubes son diseñados de acuerdo a las posibilidades de infraestructura y recursos humanos de cada escuela, dando oportunidad también a la vinculación con organizaciones públicas o privadas especializadas en aspectos educativos.

Las políticas educativas y culturales hacen especial énfasis en el reconocimiento de la diversidad cultural. Aunque la educación musical en nivel básico ha contemplado la inclusión del folclor mexicano como parte del repertorio regular, así como de otras culturas musicales, en nivel superior, los planes y programas de estudio orientados a la formación de músicos y educadores musicales siguen privilegiando la música europea de concierto (PÉREZ EXPÓSITO, 2006). Esta inconsistencia tiene implicaciones directas en la enseñanza musical, sobre el qué y cómo enseñan los egresados de licenciaturas y posgrados en música en las instituciones de educación básica. Aún parece lejos la posibilidad de concretar una adecuada difusión y apreciación de las culturas populares, indígenas, urbanas y comunitarias, como lo plantea el Plan Sectorial de Educación, cuando estas no son debidamente abordadas por educadores musicales que han recibido una formación dentro de la tradición clásica europea, o por maestros normalistas con limitada formación musical en general.

Dada la diferente formación de quienes imparten la educación musical en los distintos niveles educativos, se evidencia la necesidad de alinear, en cierta medida, los planes y programas de estudio en nivel superior, de manera que sea posible formar a los futuros maestros de música y de artes dadas las necesidades curriculares que plantea el currículo en materia de educación artística y musical en nivel básico. Debido a que existe un número limitado de programas de pregrado (licenciatura) con énfasis en educación musical (hasta el año 2015, solo había 18 programas de música en el país con un fuerte énfasis en educación musical en el país) o con un enfoque interdisciplinar en las artes (12 programas que integran las cuatro disciplinas artísticas) (GONZÁLEZ-MORENO, 2015), es prioritario que un mayor número de programas favorezcan una formación pedagógica, artística y musical, apropiada al contexto de la educación básica, ya que un gran número 
de egresados se insertarán en dicho campo laboral. En este sentido, la línea de acción 5.1.4 propuesta en el PSE sobre fortalecer la educación media superior y la educación superior en materia artística y cultural es prioritaria.

\section{Conclusiones}

En conclusión, me atrevo a decir que el incremento al acceso de una educación artística y musical de calidad es una tarea pendiente que se encuentra lejos de concretarse. La educación artística y musical ha estado presente dentro de las políticas educativas y culturales, y aunque los planes y programas de estudio nacionales anteriores y actuales la han contemplado como parte "importante" del currículo, sigue siendo considerada un área adicional o periférica al currículo, posicionada detrás de aquellas materias consideradas académicas, como Español, Matemáticas o Ciencias. Independientemente de la naturaleza política de esta y de las reformas educativas que puedan surgir en el actual sexenio, es evidente que el discurso político seguirá enfatizando la calidad e integralidad de la educación. Sin embargo, el mayor reto es pasar del discurso político a la viabilidad y efectividad de las políticas públicas en materia de educación.

Dado que el nuevo modelo educativo no se logró implementar a cabalidad, es difícil visualizar los logros o la aportación general de dicha reforma en la práctica educativa, en particular cuando justo finalizó el sexenio anterior y ha iniciado el nuevo mandato y los cambios políticos históricamente han afectado de forma negativa la continuidad y desarrollo de los programas gubernamentales. Se ha debatido ampliamente respecto a la naturaleza de la misma, si se trata de una reforma educativa o más de una reforma administrativa que pone en riesgo la situación laboral de los docentes (DÍAZ BARRIGA, 2017a, 2017b; GIL ANTÓN, 2016) y hay quienes se pronuncian abiertamente sobre esta reforma educativa, como un fracaso del anterior gobierno federal (AGUAYO, 2018) o que engañosamente se reportaron avances cuando no se acercaban las metas establecidas (VERIFICADO 2018, 2018). Lo que sí se puede dilucidar de la información aquí analizada en materia de educación artística y musical, el currículo 
presenta mínimos cambios en comparación con las propuestas curriculares anteriores (SEP, 2009, 2011), y apoya este argumento de que no se trata de una reforma de naturaleza educativa, sino más de naturaleza administrativa. Las artes siguen adoleciendo de una posición en desventaja frente al resto del currículo, y los maestros de Música y de Artes en general, al igual que los demás maestros dentro del sistema educativo, sufren de las incongruencias curriculares y de las exigencias de las autoridades educativas que establecen una evaluación docente, entre otras normativas, sin el apoyo debido para su continua capacitación y desarrollo profesional. En materia educativa, los retos que enfrenta ya el actual gobierno federal son muchos, pero el mayor de todos será pasar de las palabras a los hechos.

\section{Referencias}

AGUAYO, Sergio. Reforma Educativa fue impuesta por cúpulas de sindicatos y gobierno, es otro fracaso de Peña. Proyecto Puente, 14 mayo 2018. Disponível em:

<https://www.youtube.com/watch?v=nNrqAA_MrNs >. Acesso em: 15 mai. 2018.

CIDE, CENTRO DE INVESTIGACIÓN Y DOCENCIA ECONÓMICAS. Consulta sobre el Modelo Educativo 2016. México, D.F.: Programa Interdisciplinario sobre Política y Prácticas Educativas, CIDE, 2016. Disponível em: <https://www.gob.mx/modeloeducativo2016〉. Acesso em: 23 mar. 2018.

CONGRESO DE LA UNIÓN. (1917/2017). Constitución Política de los Estados Unidos Mexicanos (última reforma 15-09-2017). Diario Oficial de la Federación. Disponível em: <http://www.diputados.gob.mx/LeyesBiblio/pdf/1_150917.pdf>. Acesso em: 27 mar. 2018.

COORDINACIÓN NACIONAL DE DESARROLLO CULTURAL INFANTIL (2013). Conaculta niños-Alas y Raíces. Disponível em: <http://www.cultura.gob.mx/alas_raices/>. Acesso em: 27 mar. 2018.

DÍAZ BARRIGA, Ángel. (2017a). ¿El Nuevo Modelo es una verdadera Revolución

Educativa? Disponível em: $<$ https://www.youtube.com/watch?v=IOolxjjvqNw>. Acesso em: 15 maio 2018. 
DÍAZ BARRIGA, Ángel. (2017b). La Reforma Educativa: Un proceso caótico. Disponível em: <https://www.youtube.com/watch?v=AQ7goeiA2Ko>. Acesso em: 15 maio 2018.

DIRECCIÓN GENERAL DE FORMACIÓN CONTINUA, ACTUALIZACIÓN Y DESARROLLO PROFESIONAL DE MAESTROS DE EDUCACIÓN BÁSICA (s.f.). Formación continua de docentes de educación básica. Disponível em:

<http://formacioncontinua.sep.gob.mx/portal/\%2ohome.html>. Acesso em: 23 mar. 2018.

GIL ANTÓN, Manuel (2016). La Reforma "Educativa" se reduce a una evaluación.

Disponível em: <https://www.youtube.com/watch?v=7USnlqgpHUE>. Acesso em: 15 mai. 2018.

GOBIERNO DE LA REPÚBLICA. (2013). Plan Nacional de Desarrollo 2013-2018. Disponível em: <http://pnd.gob.mx/wp-content/uploads/2013/05/PND.pdf>. Acesso em: 25 mar. 2018.

GOBIERNO DE LA REPÚBLICA. (2017). Plan Nacional de Desarrollo 2013-2018: Programa Especial de Cultura y Arte 2014-2016, Avance y resultados 2017. Disponível em:

<https://www.gob.mx/cultura/documentos/logros-2017-peca>. Acesso em: 23 mar. 2018.

GONZÁLEZ-MORENO, Patricia Adelaida (2010). Students' motivation to study music: The Mexican context. Research Studies in Music Education, 32(2), 185-199.

GONZÁLEZ-MORENO, Patricia Adelaida (2015). Music teacher education in Mexico:

Current trends and challenges. In S. Figueiredo, J. Soares, \& R. F. Schambeck (Eds.), The preparation of music teachers: A global perspective (pp. 104-122). Porto Alegre:

Associação Nacional de Pesquisa e Pós-Graduação em Música.

LÓPEZ OBRADOR, Andrés Manuel. (2017). Al triunfo de MORENA en 2018 se cancelará la reforma educativa: AMLO. Boletín de prensa 00-015. Disponível em:

<http://www.amlo.org.mx>. Acesso em: 10 octubre, 2018.

MIRANDA ARROYO, Juan Carlo. (2018). Reforma educativa: El Acuerdo México-OCDE Parte II. Disponível em: <https://www.sdpnoticias.com/nacional/2018/03/26/reformaeducativa-el-acuerdo-mexico-ocde-parte-ii>. Acesso em: 8 maio 2018.

NOTIMÉRICA. (2017). La reforma educativa en México: ¿En qué consiste y por qué se protesta? Disponível em: <http://www.notimerica.com/sociedad/noticia-reformaeducativa-mexico-consiste-protesta-20151013141118.html>. Acesso em: 23 mar. 2018.

PÉREZ EXPÓSITO, Leonel. (2006). La educación musical y el aprendizaje de la diversidad cultural. Reencuentro, 46. Disponível em:

<http://www.redalyc.org/articulo.oa?id=34004608 >. Acesso em: 24 mar. 2018 
SECRETARÍA DE CULTURA (2017). México: Cultura para la armonía. Disponível em: <https://www.gob.mx/cultura/acciones-y-programas/mexico-cultura-para-la-armonia>. Acesso em: 27 mar. 2018.

SEP. Reforma Integral de la Educación Básica. Planes y programas de estudio de 1993 y 2009: Puntos de continuidad y/o cambio. México, D.F.: Secretaría de Educación Pública, 2009. Disponível em:

<http://www.tecpuruandiro.edu.mx/Calidad/pdfs/ssgc_Documentos_externos/Planes_Pr ogramas_de_Estudio_1993_2009.pdf> Acesso em: 24 mar. 2018

SEP. Plan de estudios 2011: Educación básica (1a. ed.). México, D.F.: Secretaría de Educación Pública, 2011. ISBN 978-607-081-3.

SEP. Programa Sectorial de Educación 2013-2018. México, D. F.: Secretaría de Educación Pública, 2013. Disponível em:

<http://www.sep.gob.mx/work/models/sep1/Resource/4479/4/images/PROGRAMA_SECT ORIAL_DE_EDUCACION_2013_2018_WEB.pdf>. Acesso em: 24 mar. 2018.

SEP. Foros de consulta nacional para la revisión del modelo educativo: Convocatoria. México, D. F.: Secretaría de Educación Pública, 2014a. Disponível em:

<http://www.forosdeconsulta2014.sep.gob.mx/>. Acesso em: 23 mar. 2018.

SEP. Programa Especial de Cultura y Arte 2014-2018. Diario Oficial de la Federación, 28 abr. 2014b. Disponível em:

<https://www.gob.mx/cms/uploads/attachment/file/75719/PECA-2014-2018.pdf>. Acesso em: 27 mar. 2018.

SEP. Los fines de la educación en el siglo XXI. Ciudad de México: Secretaría de Educación Pública, 2016a. Disponível em:

<https://www.gob.mx/cms/uploads/attachment/file/114503/Los_Fines_de_la_Educacio_n_ en_el_Siglo_XXI.PDF>. Acesso em: 25 mar. 2018.

SEP. El modelo educativo 2016: El planteamiento pedagógico de la Reforma Educativa. Ciudad de México: Secretaría de Educación Pública, 2016b. Disponível em: <https://www.gob.mx/cms/uploads/attachment/file/118382/El_Modelo_Educativo_2016.p df>. Acesso em: 24 mar. 2018.

SEP. Propuesta curricular para la educación obligatoria 2016. Ciudad de México: Secretaría de Educación Pública, 2016c. Disponível em: <https://www.gob.mx/cms/uploads/docs/Propuesta-Curricular-baja.pdf>. Acesso em: 24 mar. 2018.

SEP. Los fines de la educación en el s. XXI (versión final). Ciudad de México: Secretaría de Educación Pública, 2017a. Disponível em: 
<https://www.gob.mx/cms/uploads/attachment/file/207276/Carta_Los_fines_de_la_educa cio_n_final_0317_A.pdf >. Acesso em: 23 mar. 2018.

SEP. Modelo educativo para la educación obligatoria: Educar para la libertad y la creatividad (2a. ed.). Ciudad de México: Secretaría de Educación Pública, 2017b. Disponível em:

<https://www.gob.mx/cms/uploads/attachment/file/207252/Modelo_Educativo_OK.pdf>. Acesso em: 23 mar. 2018.

SEP. Aprendizajes clave para la educación integral: Plan y programas de estudio para la educación básica. Ciudad de México: Secretaría de Educación Pública, 2017c. ISBN: 978607-97644-0-1. Disponível em:

<http://www.sep.gob.mx/work/models/sep1/Resource/10933/1/images/Aprendizajes_clave _para_la_educacion_integral.pdf>. Acesso em: 25 mar. 2018.

SEP. Acuerdo número 11/05/18 por el que se emiten los lineamientos para el desarrollo y el ejercicio de la autonomía curricular en las escuelas de educación básica del Sistema Educativo Nacional. Diario Oficial de la Federación, DOF:07/06/2018. Disponível em: $<$ http://www.dof.gob.mx/nota_detalle.php?codigo $=5525413 \& f e c h a=07 / 06 / 2018>$. Acesso em: 10 out. 2018.

SINEMBARGO PERIODISMO DIGITAL. La CNTE: 34 años de historia de un organismo disidente que desafía la primera "gran reforma" de EPN. SinEmbargo Periodismo Digital, 25 ago. 2013. Disponível em: <http://www.sinembargo.mx/25-08-2013/730711>. Acesso em: 15 maio 2018.

SNTE, SINDICATO NACIONAL DE TRABAJADORES DE LA EDUCACIÓN. (2018).

Convocatoria Beca Comisión/Económica 2017-2018. 2018. Disponível em: <http://www.snte.org.mx/seccion42/vernoticias/841/o/convocatoria-becacomisioneconomica-2017-2018>. Acesso em: 15 maio 2018.

VERIFICADO 2018. La educación no ha mejorado tanto como dice el gobierno federal. Forbes Mexico, 28 mar. 2018. Disponível em:

<https://www.forbes.com.mx/verificado2018-la-educacion-no-ha-mejorado-tanto-comodice-el-gobierno-federal/>. Acesso em: 29 mar. 2018.

Recebido em: $23 / 10 / 2018$ Aprovado em: 10/12/2018

Universidade do Estado de Santa Catarina - UDESC Programa de Pós-Graduação em Educação - PPGE Revista Linhas

Volume 20 - Número 42 - Ano 2019 revistalinhas@gmail.com 